\title{
Spring-block model for a single-lane highway traffic
}

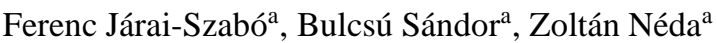

${ }^{a}$ Faculty of Physics, Babes-Bolyai University, RO-400084 Cluj-Napoca, str. Kogalniceanu nr. 1, Romania

\begin{abstract}
A simple one-dimensional spring-block chain with asymmetric interactions is considered to model an idealized single-lane highway traffic. The main elements of the system are blocks (modeling cars), springs with unidirectional interactions (modeling distance keeping interactions between neighbors), static and kinetic friction (modeling inertia of drivers and cars) and spatiotemporal disor'der in the values of these friction forces (modeling differences in the driving attitudes). The traveling chain of cars correspond to the dragged spring-block system. Our statistical analysis for the spring-block chain predicts a non-trivial and rich complex behavior. As a function of the disorder level in the system a dynamic phase-transition is observed. For low disorder levels uncorrelated slidings of blocks are revealed while for high disorder levels correlated avalanches dominates.
\end{abstract}

'Keywords: highway traffic, disorder induced phase transition, spring-block models

\section{Introduction}

Road traffic is a non-linear complex phenomenon which strongly affects our everyday life. Within this phenomenon various forms of collective behavior may be detected. The simplest possible form of traffic is the one on a single highway lane. The motion of the queue in this simple form of traffic is primarily governed by the leading car and the statistics of driving attitudes. This simple situation becomes already quite complex if the first car is moving slowly and the differences between the driving attitudes are substantial. In such situations the queue will evolve non-continuously in avalanches of different sizes, and jams of different magnitude will continuously appear. Unfortunately, such situations are common in our everyday life, so understanding and modeling it is important in order to optimize 'our society.

Traffic studies tend to discover fundamental rules in different kind of transport systems that are essential for our social and economic efficiency. Accordingly, in this field many theoretical models have been developed. The study of traffic begins early in 1935 with the pioneering work of Greenshields [1]. In '1955 Lighthill and Whitham published the oldest and most popular macroscopic model based on the theory of fluid dynamics [2]. After these early works, and motivated by the explosive increase in road traffic, an avalanche of publications in leading international journals began. These models can be classified into four categories: microscopic models, macroscopic models, cellular automata models and non-traditional models. Detailed description and analysis of such traffic models can be found in the work of Darbha et al. [3]. More recently, the nonlinear effects of small perturbations have encouraged the development of stochastic models [4] for the highway traffic. A complete review of these traffic studies can be found in the recent work of Nagatani [5], or in Kerner's book [6]. Despite of many studies in the field, due to the complexity of the problem, there are

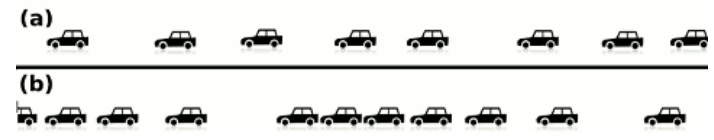

Figure 1: Traffic with laminar flow structure (a) and traffic with jams (b).

several phenomena which are still not well understood.

In order to get familiar with the complexity of the traffic and with the typical non-linear avalanche-like phenomena that can easily occur in highway traffic let us imagine that we are driving on a highway in the middle of a car queue. The traffic is normal with a laminar flow structure shown in Figure 11. Suddenly, the driver of the car located in front of us sees something and slows down a bit. To avoid collision, we should slow down only a bit, we should only reduce the foot pressure on the gas pedal. But, being concentrated to the car audio system instead we observe a little later the event and consequently we are forced to step the brakes. Seeing our braking lights, the driver behind us suddenly brakes and stops at the lane. A small event caused thus a substantial change in the traffic flow pattern. A wave consisting of cars with higher density forms and the laminar flow turns into a start-stop-start-stop motion of cars caused by temporary traffic jams that appears and propagate as sketched in Figure $1 \mathrm{~b}$.

Such situations are investigated in the present work by means of computer simulations. A simple spring-block type model with asymmetric interactions is used to model the phenomenon and the effect of parameters having major influences on the dynamics of the system will be investigated. For some model parameters interesting collective behavior is found and it is statistically studied. Up to our knowledge real experimental data describing the dynamics of cars in such idealized situation is not available. Therefore, at this stage the study has to limit itself on pure modeling, and the test of the results rely simply on our everyday experience. 


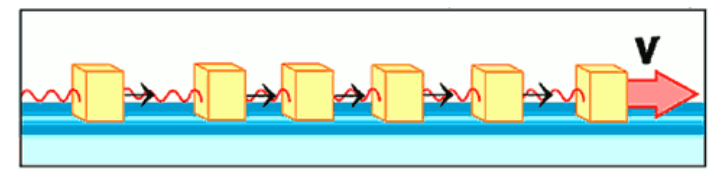

Figure 2: Sketch of the spring-block chain considered here to model the idealized single lane highway traffic.

\section{The spring-block model}

A model family with broad interdisciplinary applications is the spring-block type models. Previously we have used them successfully to study complex systems which have shown selforganization phenomena. This model family was introduced in 1967 by R. Burridge and L. Knopoff [7] to explain the empirical law of Guttenberg and Richter [8] on the size distribution of earthquakes.

This earthquake model consists of simple elements: blocks that can slide with friction on a horizontal plane connected in a lattice-like topology by springs. In the one-dimensional version of the model, the involved tectonic plates are modeled by two surfaces and their relative motion is governed by the sliding of the spring-block system on the lower surface. The upper surface (to which each block is connected by spring) is dragged with a constant velocity. Sliding of the blocks is realized by avalanches, as they are following the motion of the upper plate. These avalanches correspond to earthquakes and the energies dissipated by friction shows power law type distribution as in case of the Guttenberg-Richter law.

Subsequently, due to the spectacular development of computers and computational methods, this simple model proved to be very useful in describing many phenomena in different areas of science. Most of the collective phenomena that occurs on mezoscopic scale in solid materials can be modeled by spring-block models. The model is almost always usable when one has to deal with avalanches of different nature, complex dynamics or structure formation by collective behavior. Recently, this model has been applied successfully to explain the formation of structures obtained in drying granular materials in contact with a surface [10], to study the fractures with curved topologies in wet granular materials [11], to understand the formation of self-organized nanostructures produced by capillary effects [12, 13, 14], to study the magnetization processes and Barkhausen noise [15] and to describe glass fragmentation [16]. Based on the previous examples, one can conclude that the spring-block type models are fully applicable to the study of complex collective behavior in systems of different nature.

The spring-block model for idealized single lane highway traffic can be imagined as a chain of blocks (see Figure 2), modeling cars, connected by springs which represent some distance keeping interactions between them. This interaction represents the will of drivers to keep a certain distance from the car ahead. Therefore, in order to be realistic, these springs cannot be real, bi-directional mechanical springs because in case of traffic without accidents this distance keeping interaction acts only on the car in the back. The front car is never pulled back or pushed ahead by the car from behind unless there is a colli- sion. Accordingly, the action-reaction principle is violated and, in this sense, this spring-block system cannot be a considered as a typical mechanical system.

Another important ingredient that has to be incorporated in the model is the disorder in the system generated by the driving style of drivers. From our point of view, this should be the drivers inertia which indicates how quickly the driver can react to a certain event or how quickly can follow the velocity change of the car ahead. This is introduced via friction forces between the blocks and the plane on which they slide. These friction forces are generated randomly and independently for each new position of each of the blocks. As a first approximation we consider a normal distribution with a fixed mean $F_{m}$ and standard deviation $\sigma$. As a result of this, the disorder introduced is both spatial and temporal. Spatial disorder means differences in driving styles while temporal disorder means fluctuations of driving attitudes in time.

Moreover, in analogy with real mechanical systems static and kinetic friction forces are considered denoted by $F_{s}$ and $F_{k}$, respectively. As in usual classical mechanics systems, the static force is considered to be greater than the kinetic one, and for simplicity in our model their ratio is kept constant $f=F_{k} / F_{s}$. Here we have to note that for all the presented simulations $f=0.8$ has been used. Of course, this parameter is adjustable one and its influence on the dynamics of the system is planned to be investigated later.

Regarding the distribution of the friction forces one more comment needs to be added: when the static friction forces are randomly updated in each new position of blocks the kinetic friction force is updated, too. As a result, both the kinetic and static friction forces acting on blocks will fluctuate in time and in space.

The motion of the block queue is simulated in discrete simulation steps. For each step it corresponds the same time length $d t=1$, fixing the unit for simulation time and the possibility to handle easily the stochasticity in the equation of motions. Due to this choice we do not have possibility to define a velocity for the first block, and for us a given mean value of static friction $F_{m}$ and spring constant $k$ implicitly corresponds to a fixed drag velocity. Accordingly, the motion of the queue is governed by the drag step (the movement of the first block) which is kept constant in time. In each unit of time the first block moves ahead in steps of length $d_{0}$. In the present simulations for the spring-block row a step limit of $d_{\max }=1$ is imposed. This represents a kind of speed-limit for the units in the system. It is important to realize that by changing the drag step value we do not change the velocity of the first block. Instead of this we consider either a finer (for small $d_{0}$ values) or a rough (for large $d_{0}$ values) approximation of the continuous dynamics.

The length unit in the simulation is defined by the length of a single block $L=1$. In order to simulate the simplest dynamics without accidents, a minimum distance between blocks $d_{\text {min }}=0.3$ is imposed. This distance is considered to be also the equilibrium length $l_{0}$ of the asymmetric springs.

The motion of each block is governed by the total force acting on it. The force unit in the model has been selected by considering that all springs have the spring-constant $k=1$. Unit 
elastic force acts on the block from behind when the distance between the two blocks is $\left(d_{\min }+1\right)$ expressed in simulation length units.

With this force unit we fix the mean value of the normal distribution for friction forces $F_{m}=4$, and in the following the $\sigma$ parameter is considered to be the main parameter governing the disorder in the system.

Therefore, in this study, the model will have two free parameters: the drag step $d_{0}$ of the first block and the disorder level in the static/kinetic friction $\sigma$. All other model parameters have been fixed as $d_{\min }=0.3, d_{\max }=1.0$ and $F_{m}=4.0$ and they will not be explicitly noted in our later discussion.

Blocks are labeled after their ordinal number in the row so that the dragged block (the first in the queue) has label 1, the next one label 2, and so on until the number of blocks in the queue $N$. Their position will be noted by $x^{(i)}$, where $i=\overline{1, N}$.

The simulation follows the typical steps of a simplified molecular dynamics simulation summarized bellow.

(I) All blocks are visited and the spring force $F_{\text {spring }}$ acting on each block is calculated

$$
F_{\text {spring }}^{(i)}(t)=k\left[x^{(i-1)}(t-1)-x^{(i)}(t-1)-L-l_{0}\right] .
$$

If the block is at rest (its previous displacement is 0) the spring force is compared to the static friction. If $F_{\text {spring }}^{(i)}(t) \leq F_{s}^{(i)}(t)$ the static friction equals the spring force and the total force acting on the block will be

$$
F_{t}^{(i)}=0,
$$

and the block will remain at rest in this step. On the contrary, if $F_{\text {spring }}^{(i)}(t)<F_{s}^{(i)}(t)$ the block will start to move and the kinetic friction force is added to the spring force and

$$
F_{t}^{(i)}=F_{\text {spring }}^{(i)}-F_{k}^{(i)} \text {. }
$$

The total force is similarly calculated, when the block is not at rest (its previous displacement differs from 0 ).

(II) All blocks are visited and, based on forces $F_{t}^{(i)}$ calculated in simulation step (1), their displacements $\Delta x^{(i)}$ are calculated. The displacement of the first block is $\Delta x^{(1)}=d_{0}$ which represents the constant drag step of the first block. For the rest of blocks $i=\overline{2, N}$ the displacement is calculated by using the equation of classical mechanics with $d t=1$

$$
\Delta x^{(i)}(t)=\Delta x^{(i)}(t-1)+A F^{(i)}(t),
$$

where $A=1 / 2 m, m$ being the mass of a block. In our simulations this constant is chosen to be $A=1$ which fixes the mass of blocks to $m=0.5$ expressed in simulation units.

(III) For the calculated displacements the block step limit $d_{\text {max }}$ is applied which means, that all displacement values grater than $d_{\max }$ are set to be $d_{\max }$.

(IV) All blocks are visited following their ordinal numbers and their new positions are calculated

$$
x^{(i)}(t)=x^{(i)}(t-1)+\Delta x^{(i)}(t) .
$$

Here the only restriction is that the minimum distance $d_{\min }$ between blocks has to be respected.

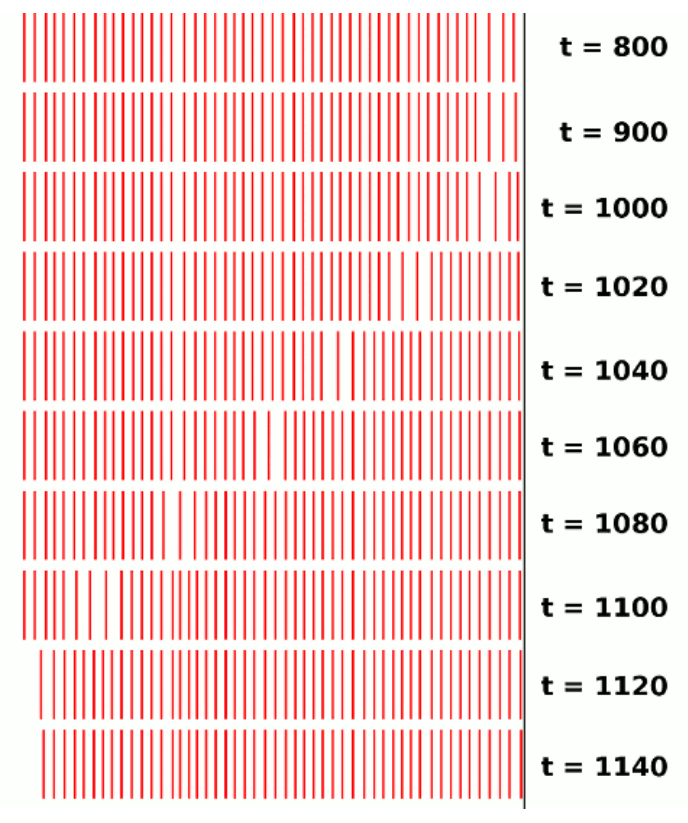

Figure 3: Time evolution of a queue with 50 blocks.

(V) Quantities of interest for the selected block are detected and stored for later analysis.

Steps (I)-(V) are repeated until enough data is collected for the statistical analyses.

\section{Results of modeling}

The dynamics of the system is illustrated with a short queue of 50 blocks in Figure 3 The first block is moving to right with small drag step $d_{0}=0.01$. The snapshots are taken on the time steps printed on images. It has to be noted that between time steps 1000 and 1100 the propagation of an avalanche through the whole system is observable.

From the viewpoint of a single block in the row it's stop-time distribution $g(\tau)$ is first measured. This stop-time distribution describes the distribution of time intervals during which a given block is not moving. In other words, this distribution function $g(\tau)$ determines the probability that the rest time of the block is $\tau$. Alternatively, the cumulative distribution function $g_{>}(\tau)$ may be defined, which gives us the probability that the stop-time of a block is bigger than $\tau$. Based on the distribution function the mean stop-time and the stop-time standard deviation may be calculated.

First, let us investigate how the position of a block in the queue is influencing its stop-time distribution. In Figure 4 the stop-time distributions of different blocks in the row are presented for a fixed small drag step $d_{0}=0.01$ and disorder in the static friction forces $\sigma=0.4$. In these simulations $N=1000$ blocks have been used and the distribution function is constructed using the statistics of 100000 stop-times. From this results it can be learned that after a certain number of blocks (transient distance), the cumulative stop-time distribution converges to the same function. Therefore, in our later investigations a block after the transient distance has to be selected in 


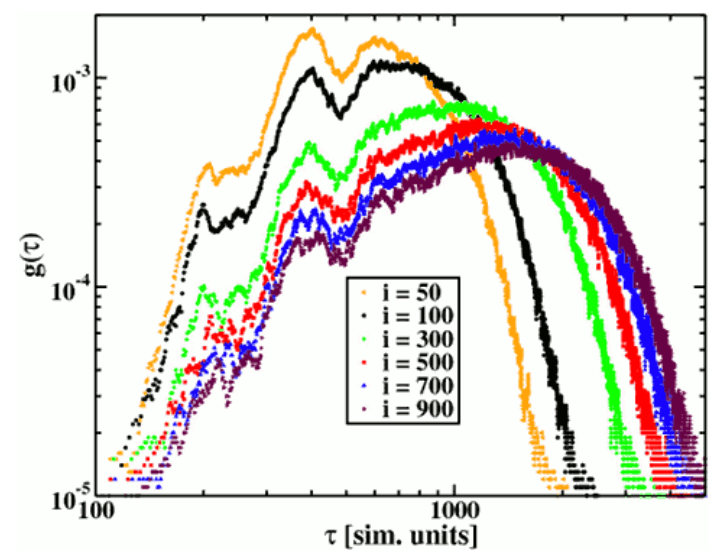

Figure 4: Stop-time distributions for different block positions $i$. The plotted results are obtained from a statistics of 100000 stop-times.

order to be sure that the average single-block behavior is studied. The form of the single block stop-time distribution function will be discussed later.

Another more illuminating analyses may be performed if one looks at the stop-time averages and standard deviations. First, their dependence on the drag step is investigated. The position of the studied block is fixed at the middle of the queue $i=500$ and several disorder levels $\sigma=0.1,0.5,1.0,1.5,2.0$ are imposed. The results are plotted in Figure 5] As one may observe, except for high drag step values the average stop-time $<\tau>$ and the standard deviation of stop-times $\Delta(\tau)$ are scaled with the drag step following a $1 / d_{0}$ functional dependence. In order to understand these scalings we have to remind ourself again, that the drag step is not equivalent with a drag velocity, and all this is a result of the fact that the simulation time-step was fixed a priori to $d t=1$. This means that if one wants to get close to a realistic, continuum dynamics, the drag step has to be lowered to infinitesimally small values. Changing the drag step is in fact equivalent to a change in the time-length $d t$ of a simulation step and thus influences only the real time of a simulation step, and implicitly everything else which is measured in time units. Therefore, this scaling suggests only that the simulations are performed in the right continuum limit. Based on our results we find that this limit is reached if drag steps smaller than 0.1 are selected.

Concerning the dynamics of the studied system interesting and non trivial results may be obtained if one looks at the influence of the friction force disorder level $\sigma$ on the stop-time averages and standard deviations. In this sense a series of simulations have been performed for several drag step values between 0.01 and 0.1 . The position of the studied block is fixed at the middle of the queue $(i=500)$, and the averages and standard deviations are calculated for 100000 stop-times. The simulation results are plotted on Figure 6 .

On one hand, as it is expected based on our previous considerations, the statistical behavior of the system for different and small enough drag steps is the same. On the other hand, in case of all curves, at a certain disorder level $(\sigma \simeq 0.7)$ a maximum in the stop-time average may be detected. This result shows us

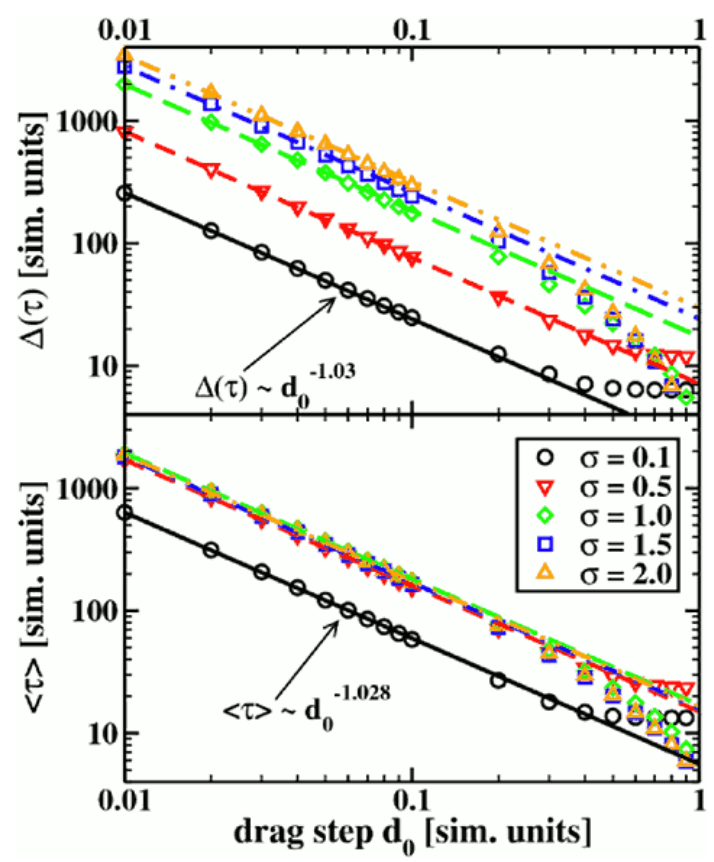

Figure 5: Stop-time averages (bottom) and standard deviations (top) depending on the drag step calculated for the 500th block in a queue of 1000 blocks. These quantities are calculated from 100000 stop-times.

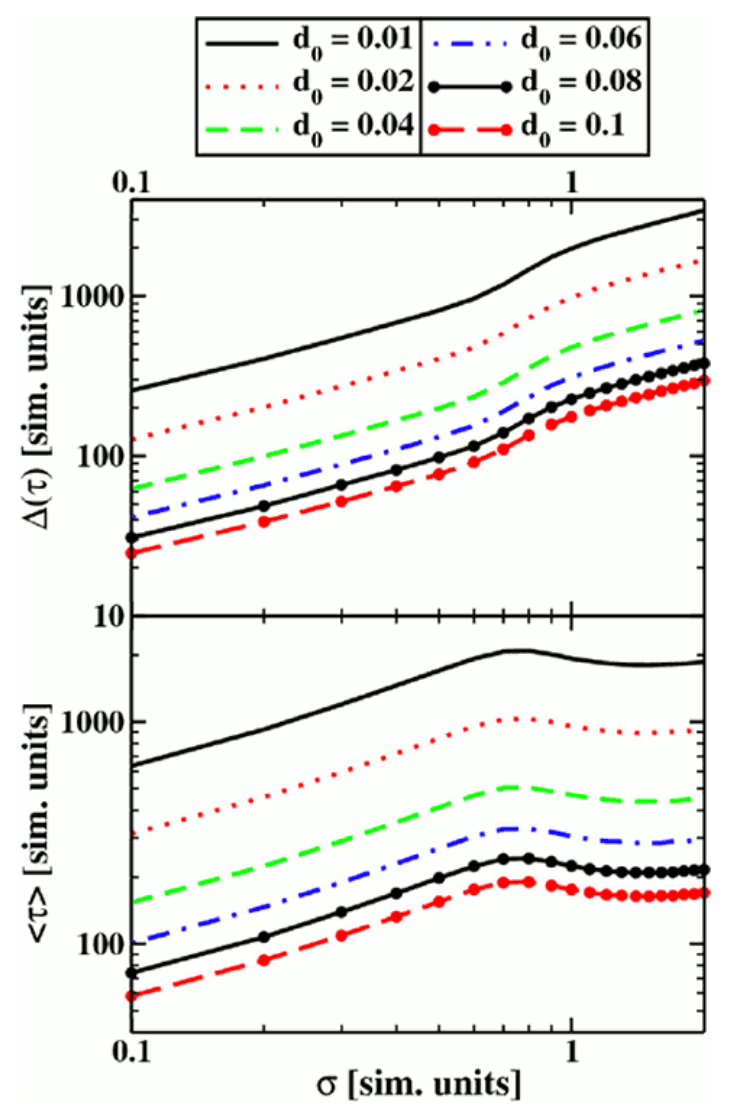

Figure 6: Stop-time averages (bottom) and standard deviations (top) depending on the disorder in static friction calculated for the 500th block in a queue of 1000 blocks. These quantities are calculated from 100000 stop-times. 
that in our simple traffic system there is a "worst" disorder level in driving attitudes (modeled by friction forces) for which the average stop-time of a block in the row is maximum. Moreover, in case of the stop-times standard deviation as a function of the mean disorder in the friction force value, at this "worst" disorder level an inflection point may be detected.

Therefore, based on these observations we can conclude that this simple spring-block system apparently exhibits two different types of behavior as a function of the disorder level in the system. At low disorder levels in the friction forces the average stop-time scales with the disorder level. This scaling law disappears however for higher disorder levels. Since this change in the system dynamics appears for a certain disorder level, we consider it as a disorder induced phase transition similar with the one recently obtained in the spring-block model of magnetization phenomena [15]. This phase transition is a high order phase transition, because a peak only in the derivative of the stop-time's standard deviation is detectable.

In order to take a closer look at the nature of this athermal phase transition detected in our spring-block system stop-time distributions of a single block are analyzed. Three disorder levels $\sigma=0.4,0.6,0.9$ are selected which correspond to values lower than, close to, and higher than the critical disorder level.

The distribution functions are constructed from 100000 simulated stop-times and they are plotted in the left panel of Figure 7 for different drag steps of $d_{0}=0.01,0.05,0.1$. As it is immediately observable, near the critical disorder level (central graph on the left panel of Figure 7 there are two peaks with the same magnitude in the stop-time distribution function best visible for drag step $d_{0}=0.01$. At lower disorder levels (top graph on left panel of Figure 7) the first peak disappears and the stoptime distribution of long stop-times will be close to a normal distribution. This may be explained if we suppose that in this region the motion of blocks is independent to each other and it is mainly influenced by friction forces generated randomly from a normal distribution. On the contrary, at higher disorder levels (bottom graph on left panel of Figure 7) the first peak, which has an exponential nature, will have the most important contribution to the stop-time distribution function suggesting stops that are induced by collective effects. It is extremely important to note that these exponential curves falls into a single one in case of different drag steps. Taking into account our previous results concerning the $\langle\tau\rangle \sim 1 / d_{0}$ scaling of average stop-times, this observation gives us an evidence that this part of the stop-time distribution is not depending on the stop-time averages and it is caused by an avalanche-like collective motion of blocks.

The same data may be analyzed from another point of view if the distribution of normalized stop-times $\tau /\langle\tau\rangle$ is constructed. This curves are plotted on the right panel of Figure 7 It is immediately observable that the Gaussian parts of distribution functions fall into a single curve. This confirms our previous presumption that the block motions at small disorder levels are mainly independent to each other which causes that the resultant distribution of normalized stop-times to be the same.

Therefore, our simulation results show that the studied spring-block system may exhibit non-trivial, critical behavior.
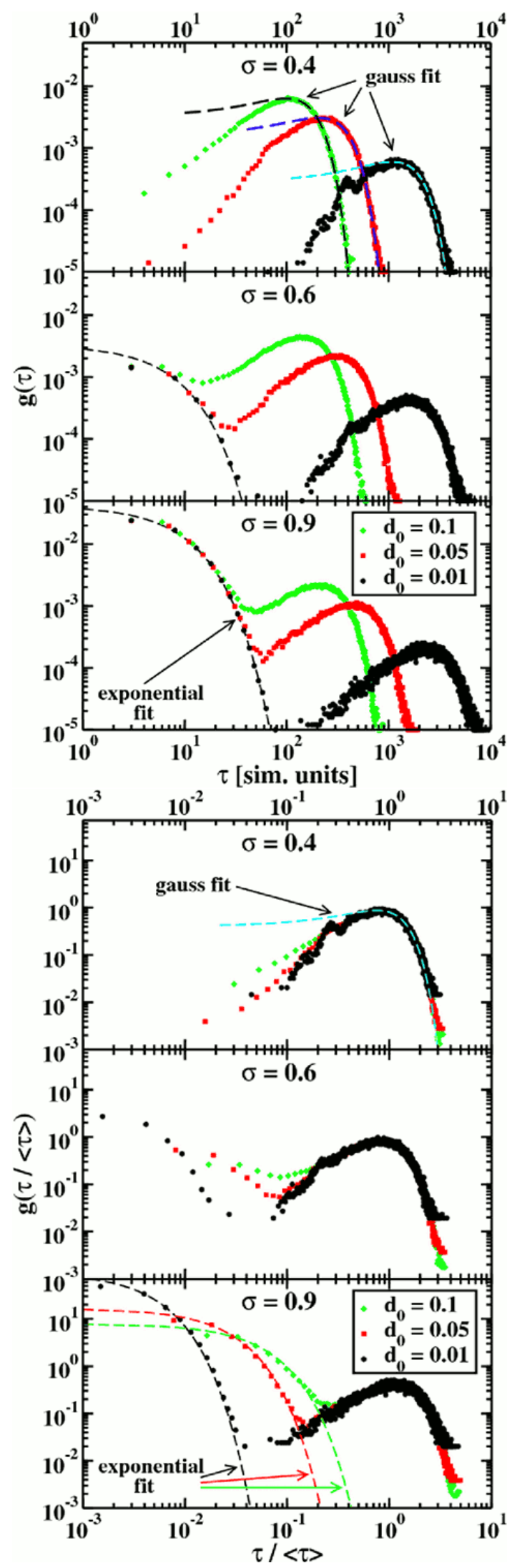

Figure 7: Stop-time distributions (top panel) and normalized stop-time distributions (bottom panel) of a single block in the middle of a queue for different disorder levels of the friction force. 
Through the detected disorder induced higher order phase transition the independent block motions will be organized to a highly correlated avalanche-like collective motion of the queue.

\section{Conclusions}

In summary, in the present paper a simple spring-block type model with asymmetric interactions has been used to model the simplest possible, idealized form of traffic which happens on one single highway lane. The spring-block chain dragged by constant steps of the leading block predicts non-trivial, complex behavior even for this simple form of traffic. Our investigations have been performed by a series of computer simulations which targeted the analysis of the effect of parameters on the model dynamics. As a result, it was concluded that at low disorder levels in friction forces the dynamics is governed by uncorrelated sliding of the blocks. On the contrary, at higher disorder levels the dynamics of the block sliding self-organizes into an avalanche-like motion of blocks. The transition between these domains is realized through a disorder induced phase transition whose effects are detectable in stop-time averages and standard deviations, too.

\section{Acknowledgments}

This work was supported by CNCSIS-UEFISCSU, project number PN II-RU PD_404/2010.

\section{References}

[1] B. D. Greenshields, Proc. Highway Research Board 14, 448 (1935)

[2] M. J. Lighthill and G. B. Whitham, Proc. R. Soc. A 229, 317 (1955)

[3] S. Darbha, K. R. Rajagopal and V. Tyagi, Nonlinear Analysis 69, 950 (2008)

[4] R. Mahnke, J. Kaupuzs, I. Lubashevsky, Phys. Rep. 408, 1 (2005)

[5] T. Nagatani, Rep. Prog. Phys. 65, 1331 (2002)

[6] B. S. Kerner, The physics of traffic, Springer, Berlin, NewYork (2004)

[7] R. Burridge and L. Knopoff, Bull. Seism. Soc. Am. 57, 341 (1967)

[8] B. Gutenberg and C.F. Richter; Ann. Geophys. 9, 1 (1956)

[9] J. M. Carlson and J. S. Langer, Phys. Rev. A 40, 6470 (1989)

[10] K.-t. Leung and Z. Néda, Phys. Rev. Lett. 85, 662 (2000)

[11] K.-t. Leung, L. Jozsa, M. Ravasz and Z.Néda, Nature 410, 166 (2001)

[12] F. Járai-Szabó, S. Astilean and Z. Néda; Chem. Phys. Lett. 408, 241 (2005)

[13] F. Járai-Szabó, A. Kuttesch, S. Astilean, Z. Néda, N. Chakrapami, P.M. Ajayan and R. Vajtai, J. Optoel. Adv. Mat. 8, 1083 (2006)

[14] F. Járai-Szabó, Z. Néda, S. Astilean, C. Farcau and A. Kuttesch, Eur. Phys. J. E 23, 153 (2007)

[15] K. Kovács and Z. Néda, Physics Letters A 361, 18 (2007)

[16] E.-A. Horváth, F. Járai-Szabó, Z. Néda, J. Optoel. Adv. Mat. 10(9), 2433 (2008) 